\title{
Télescope
}

Revue d'analyse comparée en administration publique

\section{Des grottes de Lascaux aux nuages de Google : le bouleversement des systèmes de l'écrit et son incidence sur le fonctionnement de nos institutions démocratiques}

\author{
Daniel J. Caron et Richard G. Brown
}

Volume 18, numéro 1-2, printemps-été 2012

Les administrations publiques à l'ère du numérique

URI : https://id.erudit.org/iderudit/1009261ar

DOI : https://doi.org/10.7202/1009261ar

Aller au sommaire du numéro

Éditeur(s)

L’Observatoire de l'administration publique

ISSN

1203-3294 (imprimé)

1929-3348 (numérique)

Découvrir la revue

Citer cet article

Caron, D. J. \& Brown, R. G. (2012). Des grottes de Lascaux aux nuages de Google : le bouleversement des systèmes de l'écrit et son incidence sur le

fonctionnement de nos institutions démocratiques. Télescope, 18(1-2), 155-167.

https://doi.org/10.7202/1009261ar
Résumé de l'article

En augmentant les capacités de stockage, la rapidité et la capacité de traitement de l'information, les changements opérés par la micro-informatique ont mené à des avancées dans la qualité du service aux citoyens. Les progrès plus récents ont toutefois des retombées imprévues : ils bouleversent non seulement les pratiques usuelles internes, mais remettent en question le système de l'écrit et ses conventions sur la base duquel nos bureaucraties se sont établies. Le document, instrument de première importance dans la conduite des affaires de l'État, a perdu peu à peu ses attributs et tarde à se redéfinir dans l'environnement numérique. Il en est de même pour les règles et les processus qui sont le fondement même de notre fonctionnement en organisation formelle. Cet article aborde les questions liées à l'effondrement des conventions du système de l'écrit et à la manière dont cela affecte le fonctionnement de nos institutions et requiert des innovations importantes et fondamentales si nous voulons assurer la pérennité de nos systèmes démocratiques. 


\title{
D3\$ 9r0773\$d3L4\$(4U $><4 \mathrm{U}><|\backslash| \mathrm{U} 493 \$ \mathrm{~d} 3$ 9009L3:L'3PhPh0 $\backslash \mid$ Dr3/ $/ \backslash 3|\backslash| 7$ d3\$

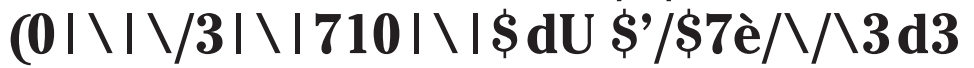 L’é(r17 37 \$0 I I 1/\P4(7 \$Ur L3 Ph0 $\backslash \mid(710|\backslash||\backslash 3 / \backslash / 3| \backslash|7 \mathrm{~d} 3| \backslash \mid 0 \$$ 1\\\$717U710|\| dé/\/0(rr471QU3\$ ${ }^{1}$
}

Par Daniel J. Caron, Administrateur général, Bibliothèque et Archives Canada et Professeur associé, École nationale d'administration publique du Québec

- danielj.caron@enap.ca

Et Richard G. Brown, Directeur général, Recherche stratégique, Bibliothèque et Archives Canada • richard.brown@bac-lac.gc.ca

\begin{abstract}
RÉSUMÉ En augmentant les capacités de stockage, la rapidité et la capacité de traitement de l'information, les changements opérés par la micro-informatique ont mené à des avancées dans la qualité du service aux citoyens. Les progrès plus récents ont toutefois des retombées imprévues : ils bouleversent non seulement les pratiques usuelles internes, mais remettent en question le système de l'écrit et ses conventions sur la base duquel nos bureaucraties se sont établies. Le document, instrument de première importance dans la conduite des affaires de l'État, a perdu peu à peu ses attributs et tarde à se redéfinir dans l'environnement numérique. Il en est de même pour les règles et les processus qui sont le fondement même de notre fonctionnement en organisation formelle. Cet article aborde les questions liées à l'effondrement des conventions du système de l'écrit et à la manière dont cela affecte le fonctionnement de nos institutions et requiert des innovations importantes et fondamentales si nous voulons assurer la pérennité de nos systèmes démocratiques.
\end{abstract}

ABSTRACT The advent of microcomputing has signified major transformations in terms of the effectiveness and efficiency of bureaucratic processes. As data storage and processing capacities have continually increased, these changes have also triggered improvements in the quality of public services. More recently, however, such progress has generated some unexpected outcomes, not only causing disruption in the customary internal practices but also throwing into question the writing system and accompanying conventions upon which our bureaucracies have been established. The leading instrument in the conduct of government affairs, the document has gradually been shorn of its attributes and has, at the same time, been slow to achieve a new definition in the digital environment. The same observation applies to the rules and processes that are the very foundation of operations in formal organizations. This article discusses the issues surrounding the collapse of the writing system and how this trend affects the operations of our institutions and compels carrying out major, fundamental innovations if we wish to safeguard the long-term future of our democratic systems.

Pour citer cet article : Caron, D. J. et R. G. Brown (2012). « Des grottes de Lascaux aux nuages de Google : le bouleversement des systèmes de l'écrit et son incidence sur le fonctionnement de nos institutions démocratiques », Télescope, vol. 18, n 1-2, p. 155-167.

1 Le titre est écrit en langage de hacking et signifie : Des grottes de Lascaux aux nuages de Google : le bouleversement des systèmes de l'écrit et son incidence sur le fonctionnement de nos institutions démocratiques. 
$\mathrm{P}$ oser la question du numérique dans le cadre de l'État revient à poser la question du fonctionnement des institutions de l'État. Le formulaire électronique et plus généralement le document électronique amenés par la micro-informatique ont certainement changé le fonctionnement interne de nos bureaucraties. De même, le rapport de l'administration avec les citoyens aussi bien que celui des représentants politiques avec leurs commettants ont eux-mêmes été bouleversés par l'informatisation (Proulx, 1984, p. 4; Valeri et Lenihan, 2003, p. 18) de leurs instruments traditionnels : les processus (Weber, 1995, p. 292) et les documents (Brosius, 2011, p. 5). Mais la première chose que nous devons noter de cette transformation initiale de l'environnement bureaucratique est que nous parlons ici de " numérisation " des rapports et non de rapports " numériques " (Caron, 2011, p. 16). Le numérique, quant à lui, représente un changement beaucoup plus profond que les transformations liées à la seule informatisation des processus, des activités et de la documentation par la micro-informatique et Internet. Le numérique dépasse le monde numérisé et ne peut être assimilé à cette seule dimension. Il entraîne des conséquences plus fondamentales : il transforme le rapport (Doueihi, 2011, p. 58) entre les individus et les processus. Il compromet - au sens de nuire, de mettre en péril - les perceptions et les anticipations habituelles des individus face aux institutions publiques sur la base desquelles nos bureaucraties ont été construites, parce qu'il affecte les comportements individuels et collectifs mais surtout, dans le thème qui nous intéresse ici, les " routines administratives " mises en place par l'administration publique pour intervenir dans la société. Il les court-circuite comme dans le cas de notre système de sécurité publique et judiciaire lorsque, par exemple, les citoyens "s'auto-incriminent " en exposant leurs exploits sur YouTube ${ }^{2}$. Nous passons ici clairement et presque directement de l'" acte répréhensible " à la Cour par " décisions » ou plus justement par pressions populaires. Dans ces cas, nous ne pouvons pas - ici l'État et son appareil - ne pas agir, ne pas réagir, ne pas intervenir même si cette manière de faire ne répond pas nécessairement aux paramètres du mécanisme bureaucratique prévu à cet effet. La population s'attend à ce que les gestes soient traités "sur-le-champ ».

Pour être à même de juger de l'importance de ces conséquences sur le fonctionnement organisationnel, institutionnel et social, il faut sortir de nos modèles fonctionnels habituels et porter un regard plus fondamental, voire anthropologique, sur la manière dont se comportent les individus dans ce nouvel environnement. De là, en dehors de ce que nous connaissons du processus judiciaire, constitutionnel ou simplement administratif de l'État moderne, nous serons mieux à même de comprendre comment le numérique est sur le point de transformer notre fonctionnement bureaucratique et institutionnel en faisant s'effondrer le système de l'écrit et ses conventions (Caron, 2011a).

Ce texte a pour objectif d'explorer l'impact du numérique sur l'évolution des systèmes de l'écrit dans le cadre du fonctionnement de l'État, de ses bureaucraties

2 Voir par exemple la vidéo II met ses excès de vitesse sur le net : il risque cinq ans : www.wat.tv/video/ il-met-ses-exces-vitesse-sur-32pnt_2eyr9_.html. Un nombre grandissant de ce genre de vidéo apparaît sur la toile. 
et, par défaut, de la société en général. Plus précisément, il sera question d'examiner comment le passage au numérique, système de " notation " au sens d'Ingold (Ingold, 2011, p. 18), modifie profondément plusieurs facettes de la société moderne dont celle du fonctionnement de l'État. Ce nouvel environnement est difficile à cerner. C'est le cas, entre autres, parce qu'il donne naissance à un contexte évolutif où les barrières que l'on croyait inamovibles tombent successivement. C'est ici que de nouvelles règles de droit, de communication et de hiérarchie, pour ne citer que celles-ci, se définissent pour " préserver " ou perpétuer sous d'autres formes les acquis des citoyens au sein des sociétés démocratiques et créer de nouveaux espaces de libertés individuelles et collectives. Dans ce creuset où se forment les prochains rapports État-citoyens semble dominer une nouvelle forme du " consigné ", de la notation, qui nous rappelle de plus en plus l'oralité mais avec, en prime, la capacité d'être préservée en masse : le numérique.

\section{— LE SYSTÈME DE L’ÉCRIT : PILIER DES INSTITUTIONS MODERNES}

Une relation étroite existe entre la nécessité de transmettre de l'information, l'apparition des techniques d'écriture, le développement de systèmes de l'écrit qui l'appuient et le développement de l'État et de la société civile. Les types de systèmes d'écriture eux-mêmes (par exemple, l'art pariétal que l'on trouve dans les grottes de Lascaux, l'art rupestre et les gravures laissées par les Premières Nations du Canada, les hiéroglyphes égyptiens et l'écriture cunéiforme) ainsi que les plateformes de communication sur lesquelles ont été faites les notations (par exemple, la pierre, l'argile, le papyrus, le papier jusqu'aux disques durs et, tout récemment, l'infonuagique) ont permis la transmission de l'information et ont soutenu la création de systèmes étatiques et la manière dont l'humain a organisé et développé les mécanismes par lesquels il arrive à fonctionner en société : les villes-États, les empires, la société mondialisée et aujourd'hui la société participative. À travers le temps, le pouvoir a évolué et s'est élargi allant des oligarchies, d'origine grecque, où il était entre les mains d'un petit groupe d'élites tels que les prêtres ou encore les rois, en passant par le rôle d'importance croissante des militaires dans la construction des empires jusqu'à l'expansion du commerce et le développement d'une classe d'administrateurs publics. L'évolution de l'enjeu de pouvoir de cette classe est bien illustrée par le système de l'écrit dans l'Égypte pharaonique tel qu'il est décrit par Posner : " [...] the overwhelming significance of record-keeping in Egypt may be traced to the fact that the functioning of its economy and its government depended on creating, preserving and having available two sets of records: the land records and the tax rolls " (Posner, 1972, p. 78).

Dans ce contexte, l'écrit est passé d'une compétence élitiste d'une grande rareté pour appuyer l'État (Caron, 2011b, p. 320; Caron et Kellerhals, 2011, p. 100) à celle de la création-expression du plus grand nombre de gens (Caron et Brown, 2012). Nous sommes ainsi passés des scribes copistes à l'autoédition dans un environnement d'informatique en nuage. Pour de nombreux spécialistes, la création, et l'utilisation de l'imprimerie, a été un événement clé dans le développement de l'écriture, non seulement parce qu'elle a augmenté la disponibilité du texte dans sa forme écrite et la disponibilité de l'information pour le citoyen ordinaire, mais 
aussi parce qu'elle a transformé, par le fait même, la relation de pouvoir entre ceux qui possédaient la capacité de lire et d'écrire et ceux qui ne la détenaient pas. Cela a réduit considérablement l'écart relationnel entre ceux qui gouvernent et ceux qui sont gouvernés. Dans l'ère numérique, cet état de fait s'est encore amplifié étant donné la capacité grandissante des citoyens de s'engager directement auprès de leurs gouvernements par la collaboration permise à travers diverses formes de technologies émergentes, mais aussi par la " sousveillance " qu'ils sont en mesure d'exercer de manière de plus en plus efficace et durable. La nouvelle littératie, dans cet environnement, a dépassé la simple capacité de lire l'écrit consigné sur un support fixe; elle prend désormais plutôt la forme d'une sorte de " translittératie " où l'individu est engagé simultanément dans une multitude de moyens de communication. Il n'est plus seulement attentiste : il participe de plein droit et très efficacement à tisser la toile des échanges communicationnels et à la création des nouveaux rapports. On parle aujourd'hui de « big sister » en contrepoids à " big brother " (Caron, 2011, p. 26). C'est ainsi que le fonctionnement des sociétés démocratiques s'est également transformé avec l'introduction des notions telles que le " gouvernement ouvert " et la " transparence gouvernementale " acceptant de moins en moins de " secrets " et réduisant davantage les relations bureaucratiques complexes. On verra que le système wébérien comme celui de la nouvelle gestion publique sont remis en question au moins pour ce qui est de la dimension processuelle des rapports entre l'État et les citoyens.

La transition dans le temps des systèmes de l'écrit centralisés à des formes décentralisées de ces systèmes et de leurs plateformes de communication a à son tour influencé directement des approches centralisées et décentralisées de l'administration publique. Pour donner un exemple simpliste, la gravure dans la pierre soutiendra des systèmes administratifs de contrôle dans de petites villes-États, alors que l'écrit consigné sur papier s'utilisera dans des systèmes administratifs décentralisés couvrant de grandes zones géographiques, et dont le rôle aura été crucial dans l'expansion du commerce et dans l'administration des empires. Il y a maintenant plus d'un quart de siècle, l'arrivée du micro-ordinateur a permis de renforcer un système encore plus décentralisé de l'administration. Aujourd'hui, avec l'informatique en nuage, le potentiel de cette administration décentralisée s'est étendu, encourageant la participation et la collaboration des citoyens dans les activités administratives et politiques de l'État. Mais il ne s'agit pas d'une simple participation accrue. C'est la nature même de cette participation qui est en train de changer. En effet, cette transition concerne directement les systèmes de l'écrit euxmêmes et leurs conventions à travers les plateformes de communication utilisées pour transmettre les messages. Mais surtout, cette transition permet graduellement aux citoyens de prendre part à la définition de ce que seront les rapports entre l'État, ses institutions et eux-mêmes forçant souvent une relation "État-citoyens " beaucoup plus bilatérale qu'unidirectionnelle. Une relation qui n'est plus simplement une relation dans laquelle le citoyen s'exprime à intervalle régulier à travers le système électoral, mais davantage une relation constante, permanente sur la manière dont l'État et sa bureaucratie se comportent, agissent au quotidien. En outre, comme le notait Harold Innis (Innis, 1950), cette interrelation entre les sys- 
tèmes de l'écrit et les systèmes d'administration a mené à une forme de monopole du savoir par l'État. Mais au fur et à mesure que la société se développe et s'étend, ce monopole est contesté par ceux qui sont à la " marge " de la société, c'est-à-dire ceux qui ne sont pas nécessairement associés à l'État ni à son administration. Ce sont ces personnes qui peu à peu participent à la création de la nouvelle forme de communication. L'invention de l'imprimerie par Gutenberg et le développement plus tard du Web par des pionniers tels que Tim Berners-Lee fournissent de bons exemples à ce cas de figure. Toutefois, avec le numérique nous remettons en question le système de l'écrit lui-même, ses fondements et ses conventions. Il devient plus difficile pour l'État de s'approprier et d'utiliser ces nouvelles formes de communication dans l'administration de la société en tentant de créer ce qui serait un nouveau monopole du savoir perpétuant le même cycle à l'origine de l'évolution des formes de l'écrit et de la communication à travers le temps.

Il y aura toujours une certaine forme de signes pour représenter la pensée et la parole. Par exemple, nous pouvons citer le cas extrême de la messagerie textuelle qui utilise des abréviations telles que " lol! ", " ur ", " 18r ", et le leetspeak, langage des technophiles et hackers qui s'exprime en caractères alphanumériques, comme nous l'avons fait pour le titre de cet article. Ces systèmes d'écriture, combinés avec les plateformes technologiques telles que la pierre, l'imprimerie ou l'informatique, ont été là pour soutenir la manifestation de l'administration publique et plus généralement ses institutions. La principale expression de cette représentation pour l'État et ses institutions a été le document.

\section{- DE L'IMPORTANCE DE CONSIGNER CERTAINS CONTENUS : ARKHEION}

Tout au long de l'histoire, le rôle de l'écrit s'est modifié selon les époques et leurs événements :

Au cours des siècles passés, [les documents] ont servi surtout à établir les obligations des citoyens envers leurs gouvernements. Cependant, depuis l'avènement des démocraties, il est devenu plus important encore de déterminer les obligations d'un gouvernement vis-à-vis de ses citoyens. Les documents doivent être créés, tenus à jour et conservés de façon à ce qu'un gouvernement démocratique contemporain puisse être tenu responsable de ses activités devant le public (Rapport de la Commission Glassco, cité par Caron, 2011c).

Ainsi, la gestion documentaire assure la responsabilisation des gestionnaires et la transparence des décisions prises et des actes posés et devient garante du fonctionnement démocratique de la société.

Le terme arkheion qui deviendra plus tard le substantif archives sera souvent confondu et assimilé au terme document. Dans tous les cas, il a un sens et un rôle fondamentaux dans le fonctionnement institutionnel qui nous interpelle directement ici. En effet, arkheion signifie " les lieux publics de la grande autorité, du grand chef ". Ce sont les lieux où l'on préserve les grands textes, les grandes lois qui guideront le fonctionnement de la Cité. Historiquement toutefois, il faut savoir que la simple création d'un contenu consigné est fondée sur une utilité précise 
et immédiate. Nous ne créons le consigné que pour servir une fin utilitaire. Ce contenu consigné deviendra l'archive et possédera ainsi, tout de suite, une valeur. Cette valeur d'utilité, c'est l'attribut principal de tout document qui serait autrement quelconque et qui permet ainsi de le nommer et de l'identifier comme étant une archive. Le document atteint un rang supérieur quand il a une valeur d'usage précise (Caron et Brown, 2012). On peut donc voir dès maintenant l'impact de la société numérique, à tout le moins sous l'angle de la surabondance de production d'information causée par une utilisation beaucoup moins contrôlée, utilitaire et paramétrée de l'écrit.

Le plus ancien texte de loi que l'on connaisse est le code d'Ur-Nammu rédigé vers 2100 av. J.-C., mais il ne nous est parvenu que de manière parcellaire. Le code de Hammourabi, texte babylonien non religieux, est l'un des plus anciens textes de loi (vers 1750 av. J.-C.) qui nous soit parvenu quasiment complet. Gravés sur une stèle en basalte, plusieurs exemplaires furent placés à travers tout le royaume dans le but de faire connaître l'autorité et la sagesse de Hammourabi sur l'ensemble du territoire qu'il dirigeait. Les « articles » du code régissaient différentes règles de la vie courante (la hiérarchisation de la société, la protection sociale notamment des esclaves, la fixation des prix, les salaires, la responsabilité professionnelle, le fonctionnement judiciaire ainsi que les peines suivant les crimes et les délits). Il s'agissait alors d'une compilation de décisions de justice, issue de la pratique jurisprudentielle et contentieuse, conservée à l'usage des générations à venir. De multiples exemples de cette nature nous parviennent par l'intermédiaire des travaux archéologiques récents (Brosius, 2011, p. 14).

Plus près de nous, depuis le Moyen Âge en Europe, les institutions chargées d'appliquer le droit ont gardé des traces écrites de leurs activités. La fonction première de ces écrits n'en était pas une de témoignage historique, mais visait plutôt à assurer la pérennité et l'authenticité des décisions prises. Par exemple, les archives des juridictions permettent d'examiner la fréquence des contentieux, la nature des litiges ou la durée des procédures. Elles permettent également de connaître les procédures utilisées et le fonctionnement des tribunaux. On peut dès lors étudier la part respective de l'écrit et de l'oral dans les procédures ainsi que le statut, la nature et les moyens de la preuve (Caron, 2011b, p. 320; Schiavone, 2008, p. 431).

Ainsi, au-delà de sa fonction d'échange et d'expression, le document joue un rôle central dans la construction de l'État de droit. Les documents appuient le fonctionnement de l'État de différentes manières : la composition de la preuve (pièce à conviction ou élément d'un dossier ayant une valeur d'évidence), le renseignement (la représentation du monde, ou le témoignage, a une valeur d'information), mais aussi le document sert à informer le citoyen sur les activités de l'État, ses institutions et sa bureaucratie. Rappelons que l'archivistique contemporaine reconnaît bien ce rôle :

Le droit d'une société ne pourrait prendre forme dans la gouvernance d'un État de droit sans cristallisation dans l'écrit : constitution, décisions politiques et juridiques, politiques publiques, comptes publics, chartes, dénombrement et caractéristiques de la population, jurisprudence et rapports en appui à la transparence se matérialisent à travers la production documentaire courante et construisent, année après année, la trame historique d'une collectivité (Caron, 2011b, p. 320). 
Tout le fonctionnement de l'État repose sur la production documentaire.

En bref, ce sont les documents, les archives dans l'ensemble de leur cycle de vie, qui permettent aux citoyens de fonctionner collectivement en démocratie et qui constituent la causa materialis de leurs organisations, de leurs entités géopolitiques et administratives. Cette causa materialis est constituée des biens civiques d'une nation : les pièces originales qui documentent les décisions et les actes, ainsi que l'information contenue dans les livres et autres médias et objets documentaires. Ces biens sont indispensables à la société pour définir, exprimer et partager ses buts communs. Ils fournissent, par ailleurs, aux citoyens et aux groupes les compétences en littératie sociale indispensables à la participation démocratique au sein de leurs collectivités (Caron, 2011c). Les processus bureaucratiques ne pourraient exister sans eux, sans les traces, sans les preuves qu'ils représentent.

\section{- LES RÈGLES ET LES PROCESSUS : L'ORGANISATION BUREAUCRATIQUE}

Autant le fonctionnement des organisations du secteur privé que du secteur public repose sur des règles et des processus. C'est l'essence même d'une organisation bureaucratique. Dans son ouvrage sur les affaires, Karlof (1990) traite des multiples processus et manières de faire qui sont le propre du secteur privé. Il y a là une même préoccupation pour la routine et la prévisibilité que dans le secteur public. Sans en faire un élément aussi central que dans le cas de la bureaucratie wébérienne, de la nouvelle gestion publique (De Visscher et Varone, 2004) ou de la science administrative classique (Gournay, 1966), Arrow montre comment le fonctionnement des organisations et des bureaucraties modernes, quel que soit leur environnement, est constitué de règles et de processus et s'appuie sur une documentation abondante. Ces éléments sont au cœur du fonctionnement organisé (Arrow, 1974). Arrow ira plus loin que la plupart des auteurs classiques en soulevant de manière très explicite la dimension moins formelle du fonctionnement bureaucratique à travers l'existence des codes non écrits. Cette codification a des conséquences importantes pour comprendre les comportements à l'intérieur des organisations. Quoi qu'il en soit, pour l'instant, notons qu'il est aujourd'hui acquis que l'État et ses institutions sont remplis de riches et de vastes compendiums de procédures qui ont pour but de permettre leur fonctionnement de manière prévisible et équitable.

Dans son chapitre sur les types de domination, Weber explique pourquoi et comment les bureaucraties doivent suivre ces processus, ces règles et les diverses normes. Il soulève l'importance de cet amalgame de règles et de procédures en rappelant que " [...] toute domination sur un grand nombre d'individus requiert normalement (pas toujours cependant) un état-major d'individus (direction administrative) c'est-à-dire la chance (normalement) assurée d'exercer une action spécifique, instaurée pour réaliser ses ordonnances générales et ses ordres concrets - individus déterminés et obéissants fidèlement [...] " (Weber, 1995, p. 285). Simon illustre cette pensée par la manifestation d'un besoin pressant de coordination " procedural and substantive ». Il définit l'expression de la coordination 
procédurale d'abord par l'existence de l'organigramme formel et ensuite par ce qu'il recèle : l'autorité et les responsabilités (Simon, 1997, p. 8). Mais comme nous l'avons soulevé précédemment, Arrow ouvre une brèche dans cette bureaucratie aux allures d'un mécanisme d'horlogerie parfaitement synchronisé en soulevant l'existence d'une panoplie potentielle de codes informels lesquels jouent aussi un rôle important dans la conduite interne et externe des affaires. Il qualifie ces mécanismes de réseaux informels : "Ethical codes and the market system itself are to be interpreted as organisations; the market system, indeed, has elaborated methods for communication and joint decision-making. As this example makes clear, the participants in organisations may be themselves organisations as well as individuals " (Arrow, 1974, p. 33). La réglementation, les règles internes et les procédures ne peuvent, à elles seules, prétendre faire office d'uniques "ingrédients " pouvant guider la conduite. Il faut socialiser les buts recherchés et se rappeler que les individus vivent aussi à l'" extérieur " de l'organisation formelle ou plus exactement de la dimension formelle du fonctionnement organisationnel. Ils sont également influencés par ce contexte extérieur et influencent à leur tour la conduite interne de ces bureaucraties. Nous ne sommes pas en vase clos ni en circuit fermé. L'ordre bureaucratique n'est pas pur et se fortifie par les multiples contingences qu'amène, entre autres, la biographie des divers individus qui participent à l'organisation.

Mais si nous sommes en organisation, bureaucratique ou autre, c'est qu'il y a des raisons de contrôler la prise de décision afin qu'elle reflète un souhait collectif ou, en termes plus économiques, parce qu'il y a défaillance de marché et que les décisions d'intérêt public ne pourraient être prises en dehors d'un collectif organisé. C'est le cas de la bureaucratie au service de l'État et de ses institutions, et c'est ce qui conduit à un certain degré de centralisation de la prise de décision avec, comme principal instrument de mise en forme, les règles et les processus. Ces règles et ces processus requièrent un autre ingrédient qui est la documentation pour les appuyer. La question fondamentale qu'apporte le numérique est : comment travailler efficacement dans une organisation de plus en plus décentralisée? Car ironiquement, on s'organise collectivement pour prendre des décisions avec l'apport de règles et de processus qui sont en quelque sorte l'assurance que ces décisions seront " contrôlées » afin de respecter ou de refléter les choix collectifs. Mais l'une des caractéristiques fondamentales du numérique est sa force centrifuge, sa capacité de créer un monde totalement atomisé, décentralisé. Dans cet environnement, quel peut être le rôle pour l'organisation formelle? Plus précisément, sous quelles conditions peut-on avoir une organisation formelle fonctionnelle? C'est-àdire où les processus, les règles et l'acte de documenter les gestes fonctionnent de manière fiable.

\section{- LE PASSAGE AU NUMÉRIQUE}

Le numérique se manifeste dans des formes concrètes. L'informatique en nuage en est certainement l'une des manifestations les plus récentes tout comme le sont les réseaux sociaux qui s'y rattachent. Brièvement, l'informatique en nuage consiste en un modèle informatique qui, par l'entremise de serveurs distants interconnectés 
par Internet, permet un accès en réseau, à la demande, à un bassin partagé de ressources informatiques configurables, lesquelles sont proposées sous forme de services évolutifs, adaptables dynamiquement et facturés à l'utilisation. Il s'agit essentiellement de mécanismes de transmission d'information et de communication à base de capacités technologiques. Mais le numérique trouve aussi sa manifestation dans des formes moins visibles, plus discrètes. Ce sont ces dernières qui nous intéressent. Ces formes plus discrètes sont issues de mécanismes de transmission d'information et de communication hyper-flexibles et permettent des transformations monumentales dans nos manières d'agir et d'interagir. C'est ici que croît notre intérêt, car c'est ce qui affecte actuellement de plein fouet le fonctionnement de nos institutions. Nous avons déjà donné quelques exemples en droit criminel en introduction. La surveillance vidéo des rues soulève les mêmes enjeux. On peut également penser aux passeports munis d'un émetteur qui permettent désormais aux autorités frontalières d'enregistrer les allées et venues des citoyens alors qu'ils passent d'un territoire à l'autre. D'autres exemples tirés du quotidien sont tout aussi éloquents. Lors de la location d'une voiture, le client peut prendre des photos de la carrosserie pour prouver son état avant la passation du contrat. Les appareils d'aujourd'hui vont ajouter à la photo des données comme l'heure, la date et les coordonnées géo spatiales précises pour parachever, d'un simple clic, un dossier complet et d'une précision inimaginable. Et que dire de ces applications qui permettent à un ou plusieurs individus de savoir en continu l'endroit exact où se trouve chacun d'eux. Pour ces quelques exemples, c'est toute l'expectative des rapports sociaux qui change : entre les citoyens eux-mêmes, entre les citoyens et les entreprises et entre l'État et les citoyens, individuels et corporatifs. Peu à peu, et depuis un bon moment déjà, nous voyons naître mais sans réelle surprise des frictions entre l'État et les citoyens et entre les citoyens eux-mêmes, car beaucoup de ces possibilités ne font pas nécessairement bon ménage avec les règles qui avaient cours dans le monde prénumérique, c'est-à-dire les conventions du système de l'écrit comme le droit d'auteur, la preuve et sa construction, la médiation et l'authenticité ou plus simplement la protection des renseignements personnels ou l'accès à l'information.

Peut-on simplement adapter nos règles, nos processus, nos lois?

Dans son passage au numérique, le document s'est radicalement transformé, et ce, sur plusieurs plans. D'abord sa forme comme objet matériel ou immatériel, son signe comme porteur de sens, mais aussi et surtout son médium comme vecteur de communication. Les avancées technologiques qui accompagnent le numérique ont apporté à la fois une plus grande capacité de traitement et une augmentation des banques de données créant, par exemple, un plus grand nombre de données personnelles. Avec le numérique, nous sommes entrés dans une phase nouvelle où la manifestation la plus évidente du changement est la perte de la stabilité du document comme objet matériel et sa transformation en un processus construit à la demande, qui ébranle parfois la confiance que l'on mettait en lui. Ainsi la fiabilité et l'authenticité du document ne vont pas de soi, comme c'était presque le cas avec son pendant analogue. Les documents naissent et voyagent beaucoup plus librement. L'information naît, vit et meurt à une vitesse prodigieuse. La 
distinction entre les types de documents (publiés et non publiés par exemple, ou encore textuels et iconographiques) tend à disparaître, car une seule chose intéresse le citoyen et c'est d'accéder au « contenu » rapidement et de la façon la plus facile et immédiate qui soit, peu importe les distinctions conventionnelles que les gestionnaires de documents avaient l'habitude d'utiliser. De nouveaux procédés (signature électronique, processus d'identification, cryptographie) entrent en ligne de compte dans la production documentaire pour essayer de garantir la "qualité " du document et protéger ainsi ses deux fonctions de preuve et de renseignement. À cela nous devons ajouter l'émergence de nouveaux phénomènes sociaux tels que le journalisme citoyen où n'importe qui peut créer, publier et commenter de l'information. L'arrivée de Wikileaks sur le marché médiatique témoigne de cette nouvelle ère où les « filtres » d'usage et les processus traditionnels de dissémination de l'information sont devenus désuets et sont de nature à changer la dynamique à la fois sociale et politique.

Mais si nous croyons que le document est en péril, c'est que nous le voyons comme un élément statique qui doit survivre à l'arrivée du numérique. Or le numérique crée de nouvelles manières de documenter et surtout d'utiliser la documentation dans de nouveaux processus qui eux font s'écrouler nos mécanismes bureaucratiques habituels. Il ne faut pas nécessairement tenter de réinventer le document ou d'enfermer le discours ou la preuve, mais plutôt essayer de comprendre la mutation de l'espace du discours qui des archives nous a mené au document et maintenant nous conduit dans un lieu sans frontières. Le "néo-contenant " du discours est aujourd'hui le nuage, mais en réalité il n'y a plus de contenant ni de document, mais des traces, des preuves de toutes formes qui circulent en dehors d'un outil de captation organisable, qui peut facilement être indexé. Comment peut-on faire fonctionner nos processus et nos règles bureaucratiques dans cet univers qui s'appuie sur le document? Ces règles et ces processus sont-ils encore possibles? Peut-on les appuyer par une documentation appropriée, authentique et valide?

Ce sont quelques-unes des questions que pose le passage au numérique pour les administrations publiques.

\section{- CONCLUSION}

Aujourd'hui, la société, l'État et ses institutions font face à bon nombre de défis engendrés par l'évolution des technologies et la mutation de l'écrit et plus vraisemblablement l'effondrement de ce système et ses conventions. Cela touche à la notion de l'anonymat, au rôle de médiation dans les organisations bureaucratiques jusqu'aux systèmes dont le judiciaire qui se mettent, malgré eux, au diapason du numérique en voyant s'écrouler leurs pratiques traditionnelles.

Max Weber avait porté devant l'opinion publique le débat sur la question de la liberté d'expression et du travail de la presse. Le principe de l'anonymat, propre aux administrations publiques, sous lequel sont publiés à son époque beaucoup d'articles de presse était bien établi. De nos jours, ce même principe se dissipe peu à peu avec les capacités technologiques actuelles qui permettent de retracer l'origine 
de chaque publication sur le Web, aussi mineure ou brève soit-elle (un commentaire sur un site journalistique ou sur un blogue, un tweet sur Twitter, etc.) en identifiant l'adresse IP de l'ordinateur émetteur de l'information, et donc en identifiant par la même occasion son auteur. Force est d'admettre dans ce contexte (plutôt transparent, dans le sens d' "imperméable ") que le fonctionnaire dans l'administration publique n'est plus confiné à l'anonymat absolu de même que tout autre acteur de la société moderne, actif sur Internet. Aussi, les individus sont de plus en plus experts en matière de recherche et d'exploitation de l'information. Avec l'écrit numérique, l'usager acquiert très tôt dans son cheminement des compétences qui lui permettent de naviguer dans les flots d'informations à sa disposition et réduisent par le fait même tout rôle de médiation que ce soit à travers le traditionnel professionnel de l'information mais aussi, et de manière beaucoup plus importante, la médiation exercée par le supérieur hiérarchique. Il n'y a plus cette nécessité d'intermédiaire pour accéder à l'information. Reprenons enfin à titre d'exemple l'évolution récente du système judiciaire. S'il était primordial de monter tout un dossier " papier " avec enquêtes, affidavits et témoignages, un nombre croissant de preuves acceptées se manifestent aujourd'hui sous forme d'enregistrements numériques - audio ou vidéo - et servent comme outil de dénonciation ou de preuve. Le système se trouve face à de nouvelles pratiques et argumentations. Grâce à l'informatique nomade (avec les téléphones intelligents, entre autres), chacun peut s'improviser témoin, détective, journaliste ou tous ces rôles en même temps, preuve audiovisuelle à l'appui.

Concrètement, l'écrit et les autres formes numériques jouissent graduellement d'un statut légal similaire au document analogue. Au Canada, au niveau fédéral, au Québec et en France, les législations s'entendent toutes sur l'admissibilité de l'écrit numérique comme le démontrent certains articles de loi $^{3}$. Bien entendu, il existe des tensions entre ces formulations législatives, les cadres juridiques et leurs applications. Il y a aussi la question centrale de la signification de la possession, de la garde ou de la responsabilité de " l'écrit numérique et son support " dans un environnement où la base géographique et territoriale du régime règlementaire ou législatif est diffuse et s'étend au-delà des frontières géopolitiques habituelles.

\footnotetext{
3 Par exemple, voir l'article 33 du chapitre 5 de la Loi (canadienne) sur la protection des renseignements personnels et les documents électroniques: " Tout ministre, ministère, direction, bureau, conseil, commission, office, service, personne morale ou autre organisme dont un ministre est responsable devant le Parlement peut faire usage d'un moyen électronique pour créer, recueillir, recevoir, mettre en mémoire, transférer, diffuser, publier ou traiter de quelque autre façon des documents ou de l'information, si aucun moyen particulier n'est prévu à l'égard de ces actes par un texte législatif. » L'article 2 du chapitre 1 de la Loi (québécoise) concernant le cadre juridique des technologies de l'information: « À moins que la loi n'exige l'emploi exclusif d'un support ou d'une technologie spécifique, chacun peut utiliser le support ou la technologie de son choix, dans la mesure où ce choix respecte les règles de droit, notamment celles prévues au Code civil. Ainsi, les supports qui portent l'information du document sont interchangeables et, l'exigence d'un écrit n'emporte pas l'obligation d'utiliser un support ou une technologie spécifique. » L'article 1316-1 du Code civil français : « L'écrit sous forme électronique est admis en preuve au même titre que l'écrit sur support papier, sous réserve que puisse être dûment identifiée la personne dont il émane et qu'il soit établi et conservé dans des conditions de nature à en garantir l'intégrité. »
} 
C'est le cœur du problème que cet article veut soulever. Peut-on simplement " numériser "- accepter les formes numériques dans le cadre des processus existants construit autour d'un monde analogue - ou doit-on innover avec, en toile de fond, ce que représentent ces nouveaux rapports numériques?

Alors que l'oralité était la norme dans bon nombre de sociétés, les systèmes de l'écrit n'ont permis d'inclure qu'une portion des citoyens dits lettrés. Force est de se demander aujourd'hui si le numérique n'est pas en train de créer une nouvelle forme d'analphabétisme bien au-delà de la fracture que crée l'existence des sans-claviers. La culture du numérique dans laquelle évolue la société nous pousse vers un changement inévitable de culture par rapport à l'écrit, un changement social, économique, administratif - qui implique la perte du contrôle exercé auparavant de façon plus systématique et ordonnée dans le monde analogue. S'il faut parler de fracture, c'est d'une fracture dans le système de consignation des dires et des discours, de la preuve et des traces dont il est question ici. Le consigné, dans ce cadre, est plus malléable, fluide - et donc plus à risque d'être falsifié. A-t-il vraiment le même statut que son pendant analogue? Le document, qui a de moins en moins les attributs pour appuyer les opérations des institutions de l'État, influence directement la pérennité et la viabilité des routines administratives telles que nous les connaissons. De surcroît, le numérique change les perceptions et les attentes des citoyens ce qui, en aval, vient aussi affecter directement la validité et la crédibilité des routines de l'administration.

Nous sommes maintenant clairement devant beaucoup plus que de simples documents électroniques. Ce qui a eu l'apparence d'une transformation purement technologique pendant longtemps prend aujourd'hui de nouvelles tournures qui nous forcent à repenser les fondements mêmes de notre fonctionnement organisationnel et institutionnel.

\section{BIBLIOGRAPHIE}

Arrow, K. J. (1974). The Limits of Organisation, New York, W.W. Norton \& Co.

Brosius, M. (2011). "Ancient Archives and Concepts of Record-Keeping: An Introduction ", dans M. Brosius (dir.) Ancient Archives and Archival Traditions: Concepts of Record-Keeping in the Ancient World, Oxford, Oxford University Press, p. 1-16.

Caron, D. J. (2011). Web HT.0 Pour une société informée : la pertinence numérique et ses défis pour les sociétés démocratiques au XXI siècle, Paris, Hermann.

Caron, D. J. (2011a). De la glaise aux puces électroniques : nouveaux systèmes de l'écrit, nouveaux types de décodages?, www.bac-lac.gc.ca/fra/bibliothecaire-et-archiviste-du-canada/ allocutions/Pages/glaise-puces-electroniques-discours.aspx (page consultée le $1^{\text {er }}$ mars 2012).

Caron, D. J. (2011b). « La production documentaire dans les administrations publiques : enjeux et pistes de solution ", dans N. Michaud (dir.), Secrets d'État? Les principes qui guident l'administration publique et ses enjeux contemporains, Québec, Presses de l'Université Laval, p. 319-338. 
Caron, D. J. (2011c). La tenue de documents au cour de la mémoire, de la responsabilisation et de l'administration publiques : l'expérience du Canada, www.bac-lac.gc.ca/fra/bibliothecaireet-archiviste-du-canada/allocutions/Pages/tenue-document-symposiumcybergouvernement.aspx (page consultée le $1^{\text {er }}$ mars 2012).

Caron, D. J. et R. Brown (2012) «Entre Voltaire et Borges : archiver, trier? La perspective du bien public ", Intermédialités, vol. 18, à paraître.

Caron, D. J. et A. Kellerhals (2011). «Supporting Democratic Values Through a Relevant Documentary Foundation: An Evolutionary Complex ", Archivaria, n 71, p. 99-134.

De Visscher, C. et F. Varone (2004). "Nouvelle gestion publique en action ", Revue internationale de politique comparée, vol. 11, n² 2, p. 177-185.

Doueihi, M. (2011). Pour un humanisme numérique, Paris, Seuil.

Gournay, B. (1966). Introduction à la science administrative, Paris, Armand Colin.

Ingold, T. (2011). Une brève histoire des lignes, Bruxelles, Zones sensibles.

Innis, H. A. (1950). Empire and Communications, Oxford, Clarendon Press.

Karlof, B. (1990). La stratégie des affaires : guide pratique, concepts et modèles, Paris, Les Presses du management.

Posner, E. (1972). Archives in the Ancient World, Cambridge, Harvard University Press.

Proulx, S. (1984). "Présentation. L'informatisation : mutation technique, changement de société? ", Sociologie et Sociétés, vol. 16, n 1, p. 3-12.

Simon, H. A. (1997). Administrative Behavior: A Study of Decision-making Processes in Administrative Organisations, New York, Free Press.

Schiavone, A. (2008). Ius, l'invention du droit en Occident, Paris, Belin.

Valeri, T. et D. Lenihan (2003). Finding our Digital Voice: Governing in the Information Age, The Crossing Boundaries Final Report and Recommendations, Ottawa, Centre for collaborative Government.

Weber, M. (1995). Économie et société, Tome I, Paris, Pocket. 